\title{
Research for health - a Sri Lankan perspective
}

Health is defined by the World Health Organisation as: "A state of complete physical, mental and social wellbeing and not merely the absence of disease or infirmity". Thus, research for health includes studies in many areas. Some of the key health issues needing research studies in Sri Lanka include, ensuring household food security, the increasing problem of non-communicable diseases, pollution related health problems, mitigating the effects of climate change on health, changing patterns of infectious diseases and health systems research, which involves financing, organizing and delivery of health services.

Ensuring household food security poses a considerable challenge. Strategies to increase food availability require more research on plant breeding to developing and promoting cultivation of high yielding, nutrient-rich varieties of cereals, legumes and starchy roots, which are resistant to extremes of weather and pests. Developing cost effective and practical methods to minimize post harvest losses is also necessary to reduce escalation of food prices. Research on development of nutrient-rich, palatable, convenience foods from less popular food products is likely to increase availability of low cost foods of high nutritional value, especially, for people living in urban areas. Undernutrition, due to reduced intake of both macronutrients and micronutrients has adverse effects on health, productivity and the quality of life. Micronutrient deficiency occurs due to decreased intake, and also due to low bioavailability. Studies on bioavailability of micronutrients in Sri Lankan diets are limited and it is necessary to identify methods to enhance absorption and improve the micronutrient status.

The prevalence of obesity is increasing in Sri Lanka and other Asian countries, which is a major factor contributing to the emerging epidemic of noncommunicable diseases such as diabetes, cardiovascular disease and cancer. Although anthropometric parameters are commonly used as markers of body fat to identify overweight or obese individuals, the global cutoff values cannot be directly applied due to ethnic differences in body fat. Hence, laboratory studies are necessary to estimate body fat content and distribution among the different age groups and develop country specific cutoff values to identify overweight or obese individuals using anthropometric parameters. Development of a surveillance framework for non-communicable diseases and identification of strategies to prevent such diseases is also essential to improve the quality of life and reduce the cost of health care.

There is increasing concern about the effects of climate change on health. Besides the direct effects of extreme weather such as death and injury, climate change etc., has many other indirect effects on health. These include a) increase in the number of food insecure households, b) changes in the population patterns of infectious disease vectors, c) increase in infectious diseass, specially, diarrhoeal diseases and d) impact on mental health of affected people. In fact, climate change is considered to be the biggest global health threat of the $21^{\text {st }}$ century and a major contributor to the global burden of disease and premature death (Costello et al., 2009). More intensive research on effects of climate change on health is an urgent necessity and requires the adoption of a multisectoral approach. Any new research programmes must take into consideration the existing research programmes and be linked to the ongoing programmes to ensure continuation.

Diseases due to pollution of air and water are major problems affecting many low and middle income countries, including Sri Lanka. Indoor air pollution is a key factor contributing to the marked increase in respiratory diseases and some types of cancer. Thus, research on factors responsible for indoor air pollution and methods to reduce these effects should lead to the development of evidence based policies to minimize indoor air pollution. The high prevalence of chronic kidney disease (CKD) in the north central province (NCP) of Sri Lanka is a major health concern, which is being 
investigated by several research groups. Contamination of ground water with chemical fertilizers, pesticides and heavy metals, snake bites, and genetic factors have been shown to be associated with CKD in the NCP. However, association does not necessarily imply causality and more rigorous well designed studies and a review of available scientific literature is necessary to determine the aetiology of CKD, which is likely to be multifactorial (Wickramasinghe et al., 2011). Research to develop and evaluate biofertilizers and pesticides from indigenous plants, which have minimal toxic effects is an urgent necessity. Also of importance are well designed studies to assess the effectiveness and safety of non-conventional additives used for water purification.

\section{REFERENCES}

1. Costello A., Abbas M., Allen A., Ball S., Bell S., Bellamy R., Friel S., Groce N., Johnson A., Kett M., Lee M., Levy C., Maslin M., McCoy D., McGuire B., Montgomery H., Napier D., Pagel C., Patel J., de Oliveira J.A.P., Redclift N., Rees H., Rogger D., Scott J., Stephenson J., Twigg ., Wolff J. \& Patterson C. (2009). Managing the health effects of climate change. The Lancet 373 (9676): 1693 - 1723.

2. Wickremasinghe A.R., Peiris-John R.J. \& Wanigasooriya K.P. (2011). Chronic kidney disease of unknown aetiology in the north central province of Sri Lanka: trying to unravel the mystery. Ceylon Medical Journal 56(4):143-146.

Sunethra Atukorala 